\title{
Incorporating psychiatric pharmacogenetics into family practice
}

\author{
"The current trial and error method for prescribing psychiatric medications to \\ patients often results in nonresponse and/or side effects, which can lead to patient \\ noncompliance or more serious complications."
}

\section{KEYWORDS: cytochrome enzymes - family practice $n$ personalized medicine - pharmacogenetic testing $=$ psychiatric medication}

Given that the bulk of medication treatment occurs in primary care, pharmacogenetic (PGx) testing within family practice is likely to be an important step for the advancement of personalized medicine. In terms of psychiatric medications, $75-80 \%$ of prescriptions are written by primary care providers. Our research study delivers and evaluates PGx testing for psychotropic drugs (antidepressants and antipsychotics) and our recent expansion to primary care clinics has been very well received by physicians and patients alike. We have demonstrated the feasibility of delivering PGx reports on a panel of CYP450 variants to family physicians in a manner that is generally understood and easily converted into actionable decisions by the physician. We expect that PGx testing carried out at the start of treatment, or for patients with problematic medication histories, will lead to more rapid clinical success with fewer side effects and complications.

\section{"Our pharmacogenetic report informs the doctor regarding the genetic status of five hepatic drug metabolism enzymes, and links each enzyme to a list of antidepressant or antipsychotic medications that are primarily metabolized by that enzyme."}

\section{Pharmacogenetics \& mental health}

Psychiatric patients usually first seek mental health treatment from their family physicians, and general estimates suggest that $75-80 \%$ of prescriptions for psychiatric medications were written by primary care providers [1]. The current trial and error method for prescribing psychiatric medications to patients often results in nonresponse and/or side effects, which can lead to patient noncompliance or more serious complications. For example, atypical antipsychotics have good efficacy in many patients, but commonly cause dangerous weight gain, leading to diabetes, then metabolic syndrome, and in some cases sudden cardiac death [2].

When the first medication fails to achieve satisfactory outcome, current treatment guidelines recommend switching the medication to an alternative that operates on a different mechanism in the brain. For example, if a patient does not respond well to citalopram (a selective serotonin reuptake inhibitor), a frequent strategy might be to switch to venlafaxine (a serotonin-norepinephrine reuptake inhibitor), then perhaps bupropion (a dopamine reuptake inhibitor), and then mirtazapine (a serotonin receptor modulator) if response is still not evident. The utilization of individualized or personalized medicine is an innovative strategy that is expected to achieve more effective therapies by basing prescriptions on a patient's specific genetic profile [3]. Early studies in this area report that PGx testing prior to prescribing antidepressant medication is associated with more effective improvements in symptoms, quicker responses to antidepressant treatment [4] and an overall reduction in healthcare utilization costs [5]. Genotype-directed treatment can help physicians differentiate between issues in drug metabolism in the liver and issues in drug targets in the brain. PGx information also equips physicians with the ability to more accurately predict the starting dose in a given patient and to avoid potential side effects due to unexpectedly high blood levels in poor metabolizer patients, for example.

Our PGx report informs the doctor regarding the genetic status of five hepatic drug metabolism enzymes, and links each enzyme to a list of

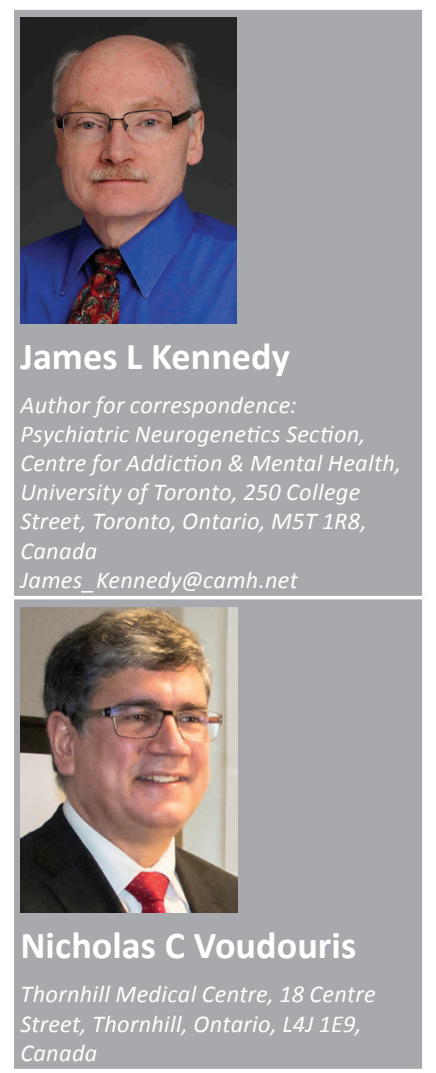

Future $\mathrm{fSS}$ 
antidepressant or antipsychotic medications that are primarily metabolized by that enzyme. The doctor can readily choose from the list of medications that a particular patient can normally metabolize based upon that patient's genetic information. In the situation wherein a patient has already failed to have good outcome on the first medication, and the genetic test results show the liver metabolism for that drug may well have been either too high or too low, the doctor can choose, if they wish, to select another medication from the same class of mechanism of action (e.g., another selective serotonin reuptake inhibitor) but that has normal metabolism gene status in that patient. In another example, if the PGx report indicates that a patient is a genetically normal (extensive) metabolizer for all potential medications, the treating physician is then able to increase or decrease the dosages to a therapeutic level with more confidence.

\section{Personalized medicine \& family practice}

Since most psychiatric patients are initially treated by their family physician, we anticipate that PGx testing will have its maximum benefit in primary care. In January 2013, we launched genetic testing of our panel of liver enzymes in a suburban Toronto family practice. Thornhill Medical Centre (TMC), in collaboration with research conducted at the Centre for Addiction and Mental Health, is the first family practice clinic in Canada, to our knowledge, to implement PGx testing into primary care for psychiatric patients.

The overall study, now conducted across multiple sites, began in June 2011. The information collected will increase our knowledge of how a person's genetic make up affects their response and side effects to psychiatric medications. The goals include, but are not limited to: identifying novel genetic and epigenetic variants associated with psychiatric drug response and side effects; integrating PGx data into electronic medical records that would be fully accessible to all physicians of a patient, and potentially having PGx reports safely accessible from mobile devices; developing more refined commercially available tests for psychiatric PGx testing; and examine if genotype-directed psychiatric drug treatment is more cost effective than the current trial and error method. To help achieve the goals above, our team is currently performing rapid genotype screening for all consenting subjects for variants in five liver enzyme genes (CYP2D6, CYP1A2, CYP2C9, CYP2C19 and CYP3A4). The results are provided to the treating physician in the form of a color-coded report, with guideline information as to which medications might be more beneficial to a given patient versus those that may have greater side effects (e.g., slow metabolizers) or lack of efficacy (e.g., ultrarapid metabolizers). Medications are displayed in the report in a three color system. Green-highlighted medications are recommended for use, whereas yellow highlighting indicates to use with caution and red highlighting means to avoid if possible. We predict this knowledge will assist physicians, in a timely fashion, to find more effective treatments for psychiatric illnesses and minimize risk for side effects.

\section{"With improved knowledge of mental illnesses and the increase in the aging population, we anticipate challenges in the management of a higher volume of patients being referred for pharmacogenetic testing in the future."}

Although our study expected obstacles in its roll-out to family practice, the experience at TMC has been well-received and successful thus far. In particular, little effort or extra time is required by the physicians to order the test in their busy schedule of seeing patients. The PGx report is produced within the second business day (produced within $48 \mathrm{~h}$ ), and easily understood by the physician. TMC was the ideal candidate for the inaugural roll-out to a family practice facility for several reasons. The Centre utilizes a state of the art electronic medical records system that is fully searchable and a complete electronic prescription system. The seven family physicians and four full-time registered nurses at TMC serve an ethnically diverse population from the Greater Toronto Area. Among the 6500 active patients served by this clinic, $5 \%$ are of African origin, 15\% are Chinese and 10\% are south Asian. Approximately a quarter of the patients are older than 65 years of age. Given the success in TMC, the next goal of our study is to expand into more family practices and eventually roll-out across the mental health and family practice departments within larger hospitals. Together, the well-trained clinical team and the efficiencies of laboratory procedures combined with TMC are helping to pave the road for personalized medicine [101].

\section{Future implications $\&$ challenges}

The implications for PGx in personalized medicine extend beyond psychiatry and an 
increasing number of primary care practitioners in the Greater Toronto Area are now seeking PGx testing in several specialized fields. The CYP2D6 enzyme, for example, has been extensively researched and even prompted detection for early risks of neurological disorders (e.g., Parkinson's disease) and cancers [6]. However, Li-Wan-Po et al. described the difficulty pharmacogenetics has faced in translating to clinical practice despite years of research [7]. Studies assessing the implementation of pretreatment PGx testing and personalized medicine have emphasized the need for the development of standards. A meta-analysis expressed the need for further validity in PGx testing, more accurate linkage between phenotype and genotypes, and prospective rather than retrospective assessments [8]. Up-to-date identification of gene variants and their phenotypic expressions for different ethnicities will be required for implementation on a global scale, and is being realized through further research.

\section{"Overall we have received a welcoming response to pharmacogenetic testing by both physicians and patients. It is evident that pharmacogenetics, as a tool for personalized medicine, is feasible, understandable and will continue to evolve over time."}

A challenge still to be met by pharmacogenetics research in all areas is in regards to polypharmacy; to find answers regarding multiple drug-drug and gene-drug interactions. Drug-herb and drug-disease interactions also pose a challenge to drug safety and personalized medicine [9]. The incorporation of drug plasma levels in the report in order to measure the phenotypes closer to the metabolizing genes, and to consider the effects of environmental/dietary factors, is warranted. Inherited or environmentally caused deficiency of an enzyme can cause accumulated levels of certain drugs in blood plasma, and vice versa for overactivated enzymatic activity. Increased toxicity may also be caused by inhibition of drug metabolism by other concurrently administered drugs [10]. Incorporating drug plasma levels will inform our analyses of interactions. Lifestyle factors, such as stress, diet and smoking, are becoming increasingly important in understanding metabolism. Grapefruit juice, for example, affects the pharmacokinetics of a variety of drugs owing to its inhibition of CYP 450
(CYP3A) enzymes [11]. Studies have also suggested inducing effects of smoking and barbequed meat on CYP450 enzymes (CYP1A2) that metabolize clozapine, olanzapine and other medications $[12,13]$.

\section{Conclusion}

Although test costs are still to be determined, PGx reports represent a lifetime of benefits for patients. Studies have started investigating the economic impact of pharmacogenetic-based treatment on healthcare utilization, and the cost burden of inadequate psychiatric medication treatments [14]. A US study recently showed significant cost savings for large and multispecialty healthcare systems [5]. Our research hopes to provide data and outcomes that would eventually lead to test approval by authorized agencies and reimbursement of PGx testing costs by healthcare providers.

Currently only a select number of genes are screened in our preliminary panel. With the addition of more genes to the PGx report, newer algorithms will be required. Presently, we are in the process of incorporating additional psychiatric medications, such as mirtazapine, bupropion and desvenlafaxine. However, the study is continuously evolving, including new genetic variants and additional medications. As the pharmacogenetic research scope expands so will the range of its clinical implications. With improved knowledge of mental illnesses and the increase in the aging population, we anticipate challenges in the management of a higher volume of patients being referred for PGx testing in the future. Overall we have received a welcoming response to PGx testing by both physicians and patients. It is evident that pharmacogenetics, as a tool for personalized medicine, is feasible, understandable and will continue to evolve over time.

\section{Financial \& competing interests disclosure}

$J L$ Kennedy has received honoraria from Novartis, Roche and Eli Lilly corporations, and he is a member (unpaid) of the scientific advisory board of AssureRx Corp. NC Voudouris has received nominal reimbursement for time spent assessing patients for their suitability for referral into the study. The authors have no other relevant affliations or financial involvement with any organization or entity with a financial interest in or financial conflict with the subject matter or materials discussed in the manuscript apart from those disclosed.

No writing assistance was utilized in the production of this manuscript. 


\section{References}

1 Mark TL, Levit KR, Buck JA. Datapoints: psychotropic drug prescriptions by medical specialty. Psychiatric Services 60(9), 1167 (2009).

2 Lett TA, Wallace TJ, Chowdhury NI, Tiwari AK, Kennedy JL, Müller DJ. Pharmacogenetics of antipsychotic-induced weight gain: review and clinical implications. Mol. Psychiatry 17(3), 242-266 (2012).

3 Maier W, Zobel A. Contribution of allelic variations to the phenotype of response to antidepressants and antipsychotics. Eur. Arch. Psychiatry Clin. Neurosci. 258(1), 12-20 (2008).

4 Hall-Flavin D, Winner J, Allen J et al. Using a pharmacogenetic algorithm to guide the treatment of depression. Transl. Psychiatry 2, e172 (2012).

5 Winner J, Allen J, Anthony A, Spahic-Mihajlovic A. Psychiatric pharmacogenomics predicts health resource utilization of outpatients with anxiety and depression. Transl. Psychiatry 3, e242 (2013).
6 Neafsey P, Ginsberg G, Hattis D, Sonawane B. Genetic polymorphism in cytochrome P450 2D6 (CYP2D6): population distribution of CYP2D6 activity. J. Toxicol. Environ. Health Part B. 12, 334-361 (2009).

7 Li-Wan-Po A. Pharmacogenetics and personalized medicine. J. Clin. Pharm. Ther. 37, 617-619 (2012).

8 Dickson R, Dundar Y, Fleeman N, Jorgensen A, McLeod C, Pirmohamed M. Cytochrome $\mathrm{P} 450$ testing for prescribing antipsychotics in adults with schizophrenia: systematic review and meta-analyses. Pharmacogenomics J. 1, 1-14 (2011).

9 Shah R, Shah D. Personalized medicine: is it a pharmacogenetic mirage? Br. J. Clin. Pharmacol. 74(4), 698-721 (2012).

10 de Leon J, Susce M, Pan R, Wedlund P, Orrego M, Diaz F. A study of genetic (CYP2D6 and $A B C B 1$ ) and environmental (drug inhibitors and inducers) variables that may influence plasma risperidone levels. Pharmacopsychiatry 40(3), 93-102 (2007).

11 Uesawa Y, Abe M, Fukuda E, Baba M, Okada Y, Mohri K. Construction of a model to estimate the CYP3A inhibitory effect of grapefruit juice. Pharmazie 66(7), 525-528 (2011).

12 Seppälä N, Leinonen E, Lehtonen M, Kivistö $\mathrm{K}$. Clozapine serum concentrations are lower in smoking than in non-smoking schizophrenic patients. Pharmacol. Toxicol. 85(1), 244-246 (1999).

13 Bibi Z. Role of cytochrome P450 in drug interactions. Nutr. Metab. 5(27), 1-10 (2008).

14 Chou W, Yan F, de Leon J et al. Extension of a pilot study: impact from the cytochrome P450 2D6 polymorphism on outcome and costs associated with severe mental illness. J. Clin. Psychopharmacol. 20(2), 246-251 (2000).

\section{Website}

101 Centre for Addiction and Mental Health: Individualized Medicine: Pharmacogenetic Assessment and Clinical Treatment. www.im-pact.ca 